\title{
Amino acid diversity in LexA repressor sequence among ESKAPE pathogens
}

\section{Rio Risandiansyah}

Microbiology Department, Medical Faculty, Univeristas Islam Malang, Indonesia

Background: Nosocomial pathogens, with the more prevalent bacteria abbreviated as ESKAPE pathogens (Enterococcus faecium, Staphylococcus aureus, Klebsiella pneumoniae, Acinetobacter baumanii, Pseudomonas aeruginosa and Enterobater), often attain antimicrobial resistance due to mutations. These mutations are acquired by SOS-response activation of which regulates over 40 different genes, including an error-prone DNA polymerase V, contributing to increased mutational frequency. SOS-response itself is triggered by DNA damage, by radical oxidative species (ROS) or other mechanisms of action of antibiotics, causing the autodigestion of LexA repressor of the SOS-box by DNA fragmentactivated RecA ( $\left.\operatorname{Rec} A^{*}\right)$ proteins. Thus, inhibition of LexA-repressor can potentially be used to prolong the life-span of current antibiotics and therefore be considered as potential drug targets for antibiotic adjuvants.

Methods: This study aims to analyze LexA-repressor in ESKAPE pathogens to determine conserved amino acid sequences to be used as a potential drug target. In this study, reference sequences of LexA-repressor was obtained from the NCBI database for ESKAPE pathogens (except for Acinetobacter baumanii due to unavailability, wherein non-reference sequences were used; $\mathrm{n}=157$ ) and was analyzed for conserved sequences using ClustalX with default setting and visualized using Skylign using weighted counts. Only LexA-repressor sequences over 190 aa were used, and putative and partial sequences were excluded. Conserved amino acids were compared with Escherichia coli $(n=36)$ to detect the presence of characteristic patterns in ESKAPE pathogens.

Results: Among the important amino acids in E. coli, as described in previous literature, were Ala-84, Gly-85, Ser-119, and Lys-156, with auto-digestion of LexA-repressor occurring between ALA-84-Gly-85 due to protease action of Ser-119. Comparison of E. coli and ESKAPE pathogens showed a conserved region of RV*AG*P**A between position 95-104 (including gaps) in ESKAPE pathogens and position 81-90 in E. coli. Similarly, Ser-119 and Lys-156 was also found to be conserved in ESKAPE pathogens compared to E. coli.

Conclusion: While LexA-repressor in ESKAPE pathogens has high variability, amino acid composition was conserved for auto-digestion which was similar to E. coli. Therefore, screening for Ala-Gly or Ser inhibitors for LexA-repressor using the current LexA-repressor molecule in $\mathrm{E}$. coli can be used to decrease resistance acquirement on multiple ESKAPE pathogens. 